\title{
Some aspects of multiparticle production in high energy nuclear interactions
}

\author{
Tufail Ahmad \\ Applied Science \& Humanities Section, University Polytechnic, Faculty of Engineering \& Technology, Aligarh \\ Muslim University, Aligarh-202002, India
}

\begin{abstract}
We report some results on mean normalized multiplicity $\left(R_{A}\right)$. This quantity shows independence with energy for pion-nucleus and proton-nucleus interactions both. The variation of $R_{A 4}$ with mean number of collisions made by incident hdaron inside the nucleus has also been studied.

Keywords: Mean normalized multiplicity, multiparticle production, created charged particles and nuclear emulsion. Subject classification PACS 13.85 - Hadron - induced high - and superhigh - energy interactions, energy $>10 \mathrm{GeV}$.
\end{abstract}

\section{Introduction}

The main concern of high energy physics is the study of fundamental particles. After having understood the properties of fundamental particles and the laws governing their interactions, one can apply these to more complex system of particles. Going back to the last many decades, one finds that there has been a growing realization that nuclear targets can give snapshots of the space-time development of a strong interaction process. Hence, it was thought that collisions of hadrons with nuclei may provide some information regarding elementary particles and their interactions which can never be known using a simple hydrogen target.

People use complex atomic nuclei in high energy experiments as targets because of the fact that it gives quite unexpected and interesting results. From theoretical point of view it is believed that at high energy a nuclear collision forces the projectile to interact with the whole lot of hadronic matter within a very short period of time. Therefore, by studying the penetration of projectile in nuclear matter, one may get some very useful informations of high energy reactions within very small distances and quite short times from impact which is definitely not possible in normal particle-particle reactions.

In the present investigation nuclear emulsion technique was used to collect the interactions/events which is when seen under a compound microscope look like a star. Nuclear emulsion is a material which memorises the tracks of charged particles. When a particle (projectile) collides with the nuclei (target) of emulsion, a large number of secondary particles are produced. There are three categories of secondary particles which are produced. First are the shower particles, they are mostly pions and are produced in forward cone, the other two are grey and black particles. The grey and black particles are also called as slow particles. The number of shower, grey and black particles in an interaction/event are termed as $\mathrm{N}_{\mathrm{s}}, \mathrm{N}_{\mathrm{g}}$ and $\mathrm{N}_{\mathrm{b}}$. When grey and black particles are taken together, they are called as heavy tracks/particles and their number is written by $\mathrm{N}_{\mathrm{h}}\left(=\mathrm{N}_{\mathrm{g}}+\mathrm{N}_{\mathrm{b}}\right)$. Many workers [1-12] have studied nuclear interactions using this technique.

In this paper we report some results on mean multiplicity, its dependence on energy and average number of collisions made by the incident particle inside the nucleus.

\section{Experimental method}

A stack of Ilford-G5 emulsion exposed to a negative pion beam of energy $340 \mathrm{GeV}$ at the CERN SPS has been used to collect the data sample. The measurement was carried out by using an oil immersion objective of 100X magnification. To make sure that data sample does not include any secondary interaction, the primaries of all the events were followed back up to the edge of the plates and only those events whose primary remained parallel to the main direction of the beam and which did not show any significant change in their ionization were finally picked up as genuine primary events. The secondary tracks coming out from each detected interaction were classified according to emulsion experiment terminology [13] on the basis of their specific ionization $\mathrm{g}^{*}\left(=\mathrm{g} / \mathrm{g}_{\mathrm{o}}\right)$, where $\mathrm{g}$ is the ionization of the track and go is the ionization of the primary. The tracks with $\mathrm{g}^{*}<1.4$, $1.4 \leq \mathrm{g}^{*} \leq 10$ and $\mathrm{g}^{*}>10$ were named as shower, grey and black tracks respectively. Other details may be found in our earlier publications [7-12].

\subsection{Mean normalized multiplicity}

\section{Experimental results}

An important parameter which may be used to understand the multiparticle production process is the mean normalized multiplicity $\mathrm{R}_{\mathrm{A}}$. The definition of mean normalized multiplicity has changed with time. 
Initially $\mathrm{R}_{\mathrm{A}}$, let us call it as $\mathrm{R}_{\mathrm{Al}}$, was defined as $\mathrm{R}_{\mathrm{Al}}=<\mathrm{N}_{\mathrm{s}}>/<\mathrm{N}_{\mathrm{ch}}>$ where $<\mathrm{N}_{\mathrm{s}}>$ represents the average number of charged shower particles observed in hadron-nucleus (hA) interactions and $\left\langle\mathrm{N}_{\mathrm{ch}}>\right.$ is the mean number of charged shower particles in hadron-hadron (hh) interactions at almost the same energy. It was realised by various workers that the above expression for the estimation of $\mathrm{R}_{\mathrm{A}}$ is not proper enough, because it was not in accordance with its definition used to predict its behaviour from various models of multiparticle production.

In emulsion experiments, only the charged particles are recorded therefore it is not possible to determine the total number of particles produced either in $\mathrm{hA}$ or in hh interactions. We, therefore, summarise here the methods proposed by various workers [14-17] to estimate the value of $\mathrm{R}_{\mathrm{A}}$ which may be compared with the predictions of various models.

\subsection{Estimation of mean normalized multiplicity in terms of actual number of final state particles} The mean normalized multiplicity in terms of actual number of final state particles is given by

$$
\mathrm{R}_{\mathrm{A} 2}=\left(3 / 2\left(<\mathrm{N}_{\mathrm{s}}>-\alpha_{\mathrm{A}}\right)+<v>+1\right) /\left(3 / 2\left(<\mathrm{N}_{\mathrm{ch}}>-\alpha_{\mathrm{H}}\right)+2\right)
$$

where $\langle v\rangle$ is the average number of collisions. $\alpha_{\mathrm{A}}$ and $\alpha_{\mathrm{H}}$ denote the leading particle multiplicities in $\mathrm{hA}$ and hh interactions respectively. Leading particle is defined as a hadron appearing in the final state and carrying the maximum share of the energy as compared to other final state particles. It is believed that the leading particle carries approximately half of the incident energy. The above expression for $\mathrm{R}_{\mathrm{A} 2}$ has been obtained on the basis of charge symmetry $[18,19] .3 / 2\left(<\mathrm{N}_{\mathrm{ch}}>-\alpha_{\mathrm{H}}\right)$ is the number of particles produced in $\mathrm{hh}$ interactions. In order to get the total number of particles in the final state, 2 has been added to account for the contribution of initial channel particles. Therefore, total expected number of particles in the final state of hh interactions is $3 / 2\left(<\mathrm{N}_{\mathrm{ch}}>-\alpha_{\mathrm{H}}\right)+2$.

Similarly, for hA interactions, $3 / 2\left(<\mathrm{N}_{\mathrm{s}}>-\alpha_{\mathrm{A}}\right)$ may be taken as actual number of particles produced. Thus to get the total number of particles in the final state, the number of particles in the initial channel, $(\langle v\rangle+1)$ should be added to it. So the average number of particles in the final state becomes $\left(3 / 2\left(<\mathrm{N}_{\mathrm{s}}>-\alpha_{\mathrm{A}}\right)+\langle v\rangle+1\right)$.

\subsection{Estimation of $R_{A}$ in terms of created charged particles}

Some workers [14-17] have taken $\mathrm{R}_{\mathrm{A}}$ as ratio of charged particles created in $\mathrm{hA}$ and hh collisions. Thus they have taken the number of charged particles created in hh collisions as $\left\langle\mathrm{N}_{\mathrm{ch}}{ }_{\mathrm{cr}}=\left\langle\mathrm{N}_{\mathrm{ch}}>-2\right.\right.$. Two has been subtracted to account for the charges present in the initial channel. For the case of $\mathrm{hA}$ collisions the number of created charged particles has been taken as $\left\langle\mathrm{N}_{\mathrm{s}}\right\rangle_{\mathrm{cr}}=\left\langle\mathrm{N}_{\mathrm{s}}\right\rangle-1$, where it has been assumed that the incident charged particle contributes one shower to the final particles and the target nucleus appear as grey. Thus, according to these workers the expression for mean normalized multiplicity becomes

$\mathrm{R}_{\mathrm{A} 3}=\left(<\mathrm{N}_{\mathrm{s}}>-1\right) /\left(<\mathrm{N}_{\mathrm{ch}}-2>\right)$

The authors in reference [14] have pointed out that while estimating the number of created charged particles, charge exchange effects should also be taken into account. In the case of pp collisions the average frequency of protons present in the final state lies between 1.27 and $1.40[18,19]$, the average of these two numbers is 1.33 . Thus the actual number of created charged particles in pp collisions may be given as $<\mathrm{N}_{\mathrm{ch}}>_{\mathrm{cr}}=<\mathrm{N}_{\mathrm{ch}}>-1.33$.

In the case of p-nucleus $(\mathrm{pA})$ interactions the incident proton appears as a proton in the final state in about sixty to seventy percent of the cases and rest of the times it appears as a neutron. Thus in order to get true number of created charged particles, 0.67 should be subtracted from the average number of particles produce in the final state. The average number of created charged particles in p-nucleus interactions may be given as, $<\mathrm{N}_{\mathrm{s}}>_{\mathrm{cr}}=<\mathrm{N}_{\mathrm{s}}>-0.67$. Therefore, in this case the expression for $\mathrm{R}_{\mathrm{A}}$, let us call it as $\mathrm{R}_{\mathrm{A} 4}$, takes the form

$\left(\mathrm{R}_{\mathrm{A} 4}\right)_{\mathrm{pA}}=\left(<\mathrm{N}_{\mathrm{s}}>_{\mathrm{cr}}\right) /\left(<\mathrm{N}_{\mathrm{ch}}>_{\mathrm{cr}}\right)=\left(<\mathrm{N}_{\mathrm{s}}>-0.67\right) /\left(<\mathrm{N}_{\mathrm{ch}}>-1.33\right)$

Similarly, for pion collisions,

$\left(\mathrm{R}_{\mathrm{A} 4}\right)_{\pi \mathrm{A}}^{-{ }_{\mathrm{A}}}=\left(<\mathrm{N}_{\mathrm{s}}>-0.50\right) /\left(<\mathrm{N}_{\mathrm{ch}}>-1.40>\right.$

\subsection{Variation $R_{\mathrm{A} 1}$ and $\mathbf{R}_{\mathrm{A} 4}$ with energy}

The values of $R_{A 1}$ and $R_{A 4}$ have been calculated using equations for $\pi^{-} A$ and $p A$ interactions. The variations of $\mathrm{R}_{\mathrm{A} 1}$ and $\mathrm{R}_{\mathrm{A} 4}$ with energy are shown in figs. 1 and 2 .

One may note from figure that $\mathrm{R}_{\mathrm{Al}}$ increases with energy up to nearly $50 \mathrm{GeV}$ and thereafter it attains an almost constant value which means that mean normalized multiplicity becomes independent of energy at higher energies. A look at the plot of $R_{\mathrm{A} 4}$ versus energy shows that $\mathrm{R}_{\mathrm{A} 4}$ is independent of energy in $\pi^{-} \mathrm{A}$ and $\mathrm{pA}$ interactions both. The average value of $\mathrm{R}_{\mathrm{A} 4}$ is $1.70+0.06$ and $1.87+0.10$ for $\pi^{-} \mathrm{A}$ and $\mathrm{pA}$ interactions respectively. This energy independent value of $\mathrm{R}_{\mathrm{A} 4}$ agrees well with the predictions of the double step models $[20-23]$. 


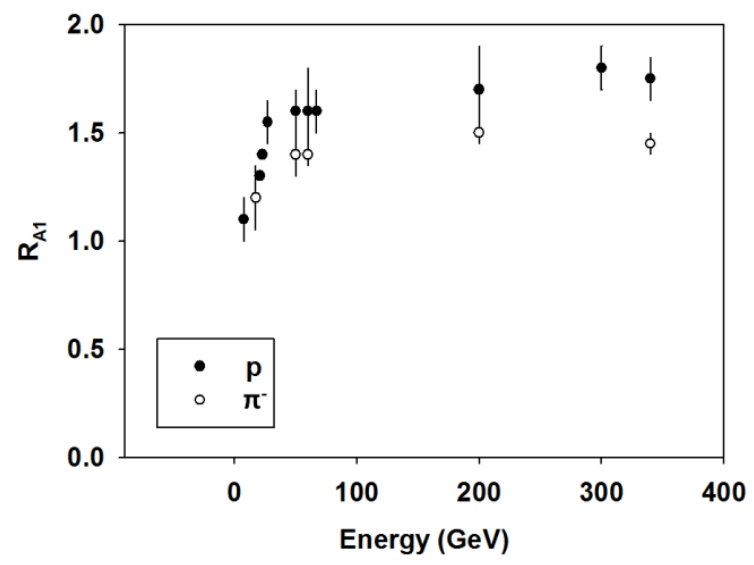

Fig. 1 Variation of $R_{A 1}$ with energy for $\pi^{-} A$ and $\mathrm{pA}$ collisions

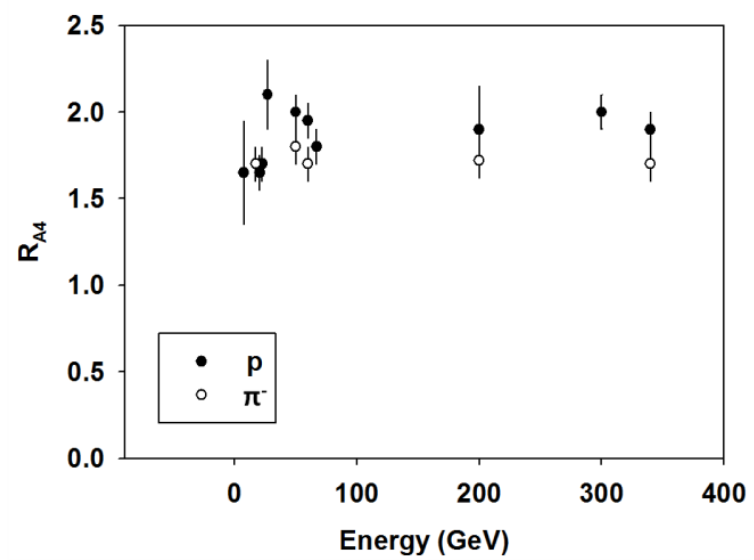

Fig. 2 Variation of $R_{\mathrm{A} 4}$ with energy for $\pi^{-} A$ and $p A$ collisions

\subsection{Variation of $\mathbf{R}_{\mathrm{A} 4}$ with the mean number of collisions}

The variation of mean normalized multiplicity with the average number of collisions, $<v\left(\mathrm{~N}_{\mathrm{g}}\right)>$ has been studied. The average number of collisions has been calculated using the method given by Stenlund and Otterlund [24]. The variation of $\mathrm{R}_{\mathrm{A} 4}$ with $\left\langle\nu\left(\mathrm{N}_{\mathrm{g}}\right)>\right.$ is shown in fig. 3. It is noted from the figure that as the values of $\left\langle v\left(\mathrm{~N}_{\mathrm{g}}\right)>\right.$ become large, an increasing trend in the value of $\mathrm{R}_{\mathrm{A} 4}$ is observed.

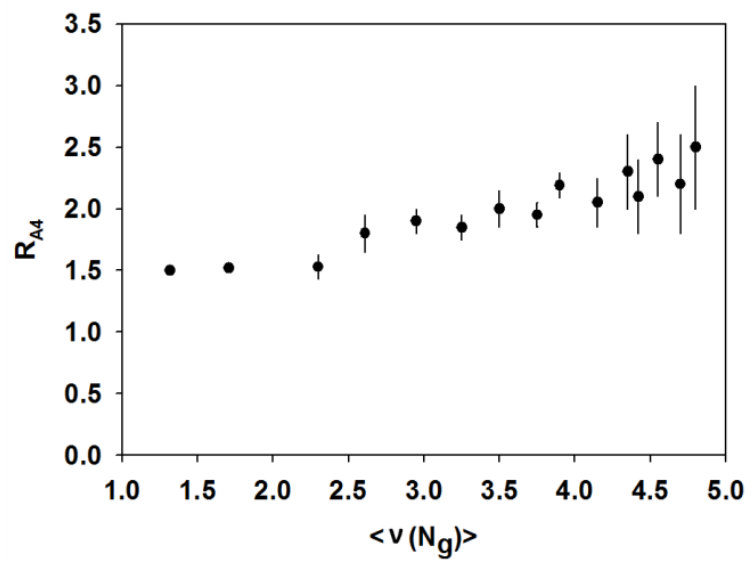

Fig. 3 Variation of $R_{A 4}$ with average number of collisions 


\section{Conclusions}

On the basis of the results presented in the paper, we conclude the following.

(i) $\mathrm{R}_{\mathrm{A} 1}$ increases with energy up to around $50 \mathrm{GeV}$ after which it attains a constant value.

(ii) $\mathrm{R}_{\mathrm{A} 4}$ is independent of energy for $\pi^{-} \mathrm{A}$ and $\mathrm{pA}$ collisions both.

\section{References}

[1]. Z. V. Anzon et al (Alma Ata-Leningrad-Moscow-Tashkent Collaboration), A study of inelastic pion-nucleus interactions at 200 $\mathrm{GeV} / \mathrm{c}$ in an emulsion, Nuclear Physics B, 129, (1977) 205-234.

[2]. E. G. Boos et al. (Alma Ata-Gatchina-Moscow-Tashkent collaboration), Investigation of inelastic interactions of $400 \mathrm{GeV}$ protons with emulsion nuclei, Nuclear Physics B, 143, (1978) 232-262.

[3]. I. Otterlund, E. Stenlund, Bo. Andersson and G. Nilson, Nuclear interactions of 400-GeV protons in emulsion, Nuclear Physics B, 142 (1978) 445-462.

[4]. M. El-Nadi, O. E. Badawy, M. K. Hegab et al, Inelastic interactions of $340 \mathrm{GeV} / \mathrm{c} \pi^{-}$with emulsion nuclei, Physical Review D, 27, (1983) 12-18.

[5]. R. K. Shivpuri and A. Kotha, Multiplicity, rapidity and rapidity correlations in $800 \mathrm{GeV}$ proton-nucleus interactions, Physical Review D, .35, 3508-3511, (1987) 3508-3511.

[6]. A. Abduzhamilov et al. (Baton Rouge-Krakow-Moscow-Tashkent Collaboration), Charged-particle multiplicity and angular distributions in proton-emulsion interactions at $800 \mathrm{GeV}$, Physical Review D, 35, (1987) 3537-3540.

[7]. A. Tufail, S. Ahmad, A. R. khan, M. Zafar and M. Shafi, Nuclear interactions of 340-GeV pions in emulsion, Physical Review D, $42,(1990)$ 2187-2193.

[8]. A. Tufail, S. Ahmad, M. Zafar and M. Shafi, Multiplicity correlations and KNO scaling in pion-nucleus interactions, Il Nuovo Cimento A, 104, (1991) 145-150.

[9]. A. Tufail, S. Ahmad and M. Zafar, Features of forward-backward multiplicity correlations in hadronic collisions, Canadian Journal of Physics, 74 (1996) 141-143.

[10]. T. Ahmad, N. Ahmad, S. Ahmad and M. Zafar, Characteristics of compound multiplicity in hadron-nucleus collisions, Indian Journal of pure and Applied Physics, 48 (2010) 855-859.

[11]. T. Ahmad, A study of pion-nucleus interactions in terms of compound particles, ISRN High Energy Physics, 2014 (2014) 1-6.

[12]. T. Ahmad, Scaling of multiplicity distribution in high energy hadron-nucleus interactions, Accepted, To appear in IOSR journal of Applied Physics, (2014).

[13]. W. H. Barkas, Nuclear Research Emulsions, Academic Press, New York, NY, USA, (1963).

[14]. T. Aziz, M. Zafar, M. Irfan, A. Ahmad and M. Shafi, Analysis of multiparticle production data on proton-nucleus collisions using a new variable, Pramana, 11 (1978) 323-332.

[15]. J. Babecki, B. Furmanska, R. Holynsky, A.Jurak, G. Nowak, H. Wilczynski, W. Wolter and B. Wosiek, Interactions of pions and protons with emulsion nuclei at $60 \mathrm{GeV}$ and $200 \mathrm{GeV}$, Acta Physica Polonica B, 9, (1978) 495-501, ibid, Angular distributions in proton-nucleus collisions at $67 \mathrm{GeV}$ and $200 \mathrm{GeV}$, Acta Physica polonica B, 5, (1974) 315-329.

[16]. V. Kumar, A. P. Sharma, M. Kaur, J. M. Kohli, I. S. Mittra and P. M. Sood, Study of charged particle multiplicity in 50 GeV/c piemulsion interactions, Journal of Physical Society of Japan, 44,(1978) 1078-1082.

[17]. D. P. Goyal, P. K. Sengupta and S. Singh, Study of R(3)-<NU> relationship from $400 \mathrm{GeV}$ p-nucleus interactions, Physical Review D, 29, (1984) 154-156.

[18]. H. Boggild, K. H. Hansen and M. Suk, On the production spectrum of pions in high energy proton-proton collisions, Nuclear Physics B, 27, (1971) 1-12.

[19]. V. V. Ammosov et al: A study of $\pi^{+}, \pi^{-}$and proton production in pp interactions at $69 \mathrm{GeV} / \mathrm{c}$, Nuovo Cimento A, $40,(1977) 237$.

[20]. A. Dar and J. Vary, Method to distinguish between multiparticle production mechanisms, Physical Review D, 6, (1972) 2412-2417.

[21]. P. M. Fishbane and J. S. Trefil, Hadronic scaling in the scattering of composite systems, Physical Review D, 8, (1973) $1467-1485$.

[22]. G. Berald, A. Dar and G.Eilam, Multiparticle production in particle-nucleus collisions at high energies, Physical Review D, 13, (1976) 161-165.

[23]. M. Ta-Chung, Multiparticle production in high energy hadron-nucleus collisions, Physical review D, 15, (1977) 197-205.

[24]. E. Stenlund and I. Otterlund, On the slow particle production in hadron-nucleus interactions, Nuclear physics B, 198, (1982) 407426. 